\title{
EL DESARROLLO SOSTENIBLE Y EL FUTURO DE LA ENSEÑANZA DE LAS CIENCIAS
}

\author{
SAEZ, M. J.' y RIQUARTS, K. ${ }^{2}$ \\ ${ }^{1}$ Universidad de Valladolid, España. \\ 2 Institute for Science Education, Kiel, Alemania.
}

\section{SUMMARY}

Although the idea of sustainable development came up in the first reports of the Club of Rome some twenty years ago, it was not taken into account by educationalists until the late eighties. The paper discusses two issues that have emerged from that of sustainable development: $a$ ) the operative consequences of this concept for citizens; $b$ ) the consequences for the educational system and curriculum. The consequences are discussed at three levels: $a$ ) the curriculum, where not only the scientific and technological innovations may be examined on environmental terms, but also the idea about economic development and growth (they are not always together on historical terms), b) on hands teacher training promoting evaluation linked to on the scene teacher training as an important skill; c) innovative learning where anticipating global situations and participating locally is based on situated learning.

\section{EL DESARROLLO SOSTENIBLE: CONSECUENCIAS OPERATIVAS}

La idea de desarrollo sostenible aparece ya en los primeros informes del Club de Roma (Meadows, 1972); las distintas formulaciones que desde entonces han surgido se fundamentan en tres ideas básicas que configuran el modo de pensar de aquéllos que lo mantienen y que son:

- La especie humana forma parte de la naturaleza, dependiendo su existencia, en gran medida, de su capa. cidad para mantener el equilibrio del mundo natural. No debemos agotar sus recursos, que son finitos, para lo cual se debe frenar el paulatino deterioro de la naturaleza y desarrollar, a su vez, sistemas de regeneración.

- La actividad económica debe incluir en los gastos de producción los necesarios para la conservación y la regeneración del mundo natural. Porque nuestro bienestar, el desarrollo actual, no pude hacerse a costa del de nuestros descendientes.

- El nivel global de calidad del medio ambiente depende del desarrollo mantenible en el tiempo que sea capaz de establecer la familia humana en su totalidad.
Estas ideas aunque no entraron a formar parte del mundo educativo hasta mediados de los 80 (Uzwyshyn, 1990), tuvieron dos consecuencias importantes en la forma de pensar. Primero, al reconocer que la planificación, en cualquier ámbito, debe hacerse a más largo plazo y con una perspectiva de globalidad; y, segundo, que es necesario evaluar permanentemente el impacto no sólo de las diferentes políticas sino también de las acciones de los seres humanos.

Pero para que estos principios consigan implantarse o simplemente comiencen a difundirse de forma más amplia, es necesario convertirlos en términos operativos, que permitan, por un lado, poder estudiarlos mejor $y$, por otro, poder adaptarlos a contextos particulares. Para ello podemos agruparlos en dos niveles de concreción, a saber:

- En el primer grupo se pueden situar los que se refieren a cuestiones de tipo ético, tales como integración, reparto, responsabilidad compartida, prevención y conservación; lo que significa que se debe gestionar la economía 
y los recursos naturales en beneficio no sólo de las generaciones presentes sino también de las futuras. Esto nos conduce a ser vigilantes del necesario equilibrio entre las decisiones de hoy y el impacto de mañana. Sólo promoviendo un espíritu de solidaridad, compañerismo y cooperación, que se apoye en cl conocimiento de cómo se toman las decisiones económicas y los efectos de su implementación se podrán mitigar y en su caso modificar las políticas que han conducido a tales decisiones.

- El segundo grupo es más operativo y concreto, porque marca pautas de actuación (Marathé, 1994) en plazos próximos, tales como el reciclaje, el estímulo, la rehabilitación, la innovación científica y tecnológica y la responsabilidad entendida de forma global. El principio del reciclije significa promover políticas de reutilización de materiales para reducir el problema de los xesiduos solidos, consumiendo por ello menores cantidades de materiales brutos, reduciendo la cantidad de recursos utilizados y disminuyendo la generación de desperdicios en la manufactura de productos, promoviendo simultáneamente la implantación de industrias que manipulen dichos residuos. Son esenciales, en cualquier caso, actuaciones encaminadas a reparar el daño realizado y la degradación del medio ambiente producida. Pero, como base importante de todo este grupo, se encuentra, sin duda, la iclea de la innovación científica y tecnológica, que debe encaminarse a mantener el equilibrio del planeta como urt ecosistema global, lo que conduce a concebir la responsabilidad en têrminos globales (Brophy, 1991), Pensar globalmente y actuar localmente de forma coordinada y coherente significa que los ciudadanos comprendan la importancia de la conexión que existe entre el medio ambiente, la salud humana y la economía. Es fundamental para ello hacer hincapié en la no existencia de fronteras en la naturaley y la interdependencia ecológica entre las regiones y naciones.

El modelo curricular más coherente con lo anteriormente planteado es, según Holbrook y Chisman (1990), un currículo que incluya valores, procedimientos y conocimientos:

- Valores en los que se enfatice el respeto por todas las formas de vida (biodiversidad), el ascetismo en cuanto al consumo de to natural, y alternativas éticas en cuanto a las prioridades sociales y culturales. Los valores deben formar parte de los objetivos educativos propiamente.

- Procedimientos que incluyan una amplia gama de estrategias de enseñanza-aprendizaje, especialmente aquéllas que enfaticen los procesos de toma de decisiones y el trabajo colaborativo, así como las claves de cómo se produce la comunicación y la participación.

- Conocimientos que deben incluir aspectos estructurales básicos y los pasos y datos obtenidos que han conducido a su descubrimiento, medio ambientales, culturales, económicos y sociales, así como la interconexión de los sistemas, la metodología que estimula los procesos científicos y tecnológicos y la manipulación del riesgo.
Resumiendo, las consecuencias del concepto de desarrollo sostenible para la enseñanza de las ciencias son de gran importancia dado que promueve que la información sea necesaria y útil, facilitando que la población pueda conocer las posibilidades y oportunidades que la ciencia aporta para una mayor calidad de vida (Riquarts, 1987; Nachtigall, 1988), cuyo planteamiento incluye que (...la presión de los grupos de poder no conduzca al abuso del conocimiento científico. La alfabetización científica, tanto personal, como social y cultural, es esencial [...] si el abuso de la ciencia debe ponerse de manifiesto, y porque los estudiantes deben ser educados para labrar su propio camino en el mundo moderno del trabajo y en su vida cotidiana, para lo cual deben ser ciudadanos informados.» (Power, 1990, 16)

\section{CONSECUENCIAS DE LA IDEA DE DESARROLLO SOSTENIBLE EN EL MUNDO EDUCATIVO}

De lo anteriormente expuesto se pueden concluir principios importantes que deben guiar la planificación educativa, destacando entre ellas la necesidad de saber y comprender que no existen barreras en nuestro medio ambiente y que se produce una inevitable interdependencia entre las naciones a todos los niveles, lo que nos conduce a pensar en términos de globalidad y de actuación a nivel local.

En términos formativos, los sistemas educativos son canales inevitables para la diseminación de ideas y de formas de contribuir a la participación activa. La participación necesita de credibilidad y transparencia desde el punto de vista político y al mismo tiempo de comprensión y profundo entendimiento de la complejidad de la situación con la que estamos tratando.

Se ha argumentado que la enseñanza de las ciencias debe ser relevante para el estudiante, pero el propio concepto de relevancia necesita de una precisa clarificación. La relevancia debe entenderse en función del papel de los individuos en la sociedad, de sus capacidades y del contexto en el que están ubicados. Cada vez más en la sociedad actual se han de tomar decisiones, algunas de las cuales tienen un ámbito de aplicación que afecta a todos los ciudadanos, en las que se hace imprescindiblo tomar en consideración el punto de vista de la ciencia y de la tecnología. Como ejemplo de ello podemos recordar los temas relacionados con reproducción asistida o terapia génica, cuyas repercusiones tienen un ámbito de aplicación tanto individual como colectivo, presente ya como debates públicos en muchos países $\mathrm{y}$, sin embargo, todavía no en otros con la misma dimensión, independientemente de lo acuciante del problema demográfico. El currículo de ciencias, por ello, debe variar según los diferentes grupos de estudiantes; el conocido lema y objetivo de «ciencias para todos» debe significar diversificación de diseños en los programas. 
Educar para satisfacer las necesidades humanas puede discutirse, por tanto, desde tres puntos de vista:

a) para el currículo, donde la idea de las innovaciones científicas y tecnológicas se examinen no sólo en términos de medio ambiente sino también en cuanto a crecimiento y desarrolio económico (que no siempre han evolucionado conjuntamente a lo largo de la historia);

b) para la formación del profesorado, incorporando a ella la evaluación como un aspecto de importante valor formativo; $y$

c) para el aprendizaje, teniendo en cuenta situaciones globales pero orientado a formas de actuación local para que se produzca aprendizaje «situado».

\section{EL CURRÍCULO DE CIENCIAS}

Actualmente el énfasis de la modernización (tanto en los países en vías de desarrollo como en los desarrollados) está en la tecnologización industrial a través del desarrollo y en la innovación, mientras que, a través de los sistemas educativos, se insiste y refuerza el correspondiente conocimiento científico y tecnológico, su competencia y sus destrezas, siendo esto lo que configura en gran medida los actuales currículos de ciencias de la mayoría de los países.

Estos objetivos de sobra conocidos han sido tratados de diferentes maneras durante las pasadas décadas, bien introduciendo nuevas asignaturas (tecnología, por ejemplo), abandonando la separación de las asignaturas tradicionalmente incluidas bajo el epígrafe de ciencias para de esta manera poder abordar la complejidad de la situación que en estos momentos se plantea, o incluyendo, bajo formas distintas, cuestiones relativas al medio ambiente. La siguiente etapa consistió en la introducción de cuestiones relativas al impacto social de la ciencia en el currículo, habitualmente reseñado con la etiqueta de currículo «ciencia, tecnologia y sociedad» (CTS). Las razones para la inclusión de este enfoque han sido ampliamente representadas por autores tales como Yager (1992), Black (1993), Cross (1993) y Anderson (1994).

En este apartado querríamos, sin embargo, centrarnos en ofrecer sólo aquellas posibilidades que aparecen en los currículos de ciencia y tecnología, y que incluyen cuestiones sobre su impacto, en el equilibrio entre las diferentes regiones del planeta y que plantean innovaciones que corresponden a necesidades humanas (Kortland, 1992; Hale, 1993). El término evaluación del impacto, en este ámbito, significa que la elección de contenidos no puede estar ya relacionada sólo con su relevancia en términos científicos, sino orientada a la capacidad, de un cierto contenido, de preparar a los estudiantes para tratar con un problema relevante.

Para clarificar esta idea, lo más adecuado parece utilizar un ejemplo de cómo estas unidades curriculares debe- rían estructurarse; usaremos una de las incluidas en un libro de reciente publicación (Riggs et al., 1993), Plásticos Biodegradables.

La unidad ofrece información sobre una variedad de temas que pueden agruparse en cuatro áreas:

a) La unidad provee del conocimiento químico previo sobre plásticos (polimeros de carbono) e información básica sobre compuestos orgánicos y carbohidratos y cómo las bacterias pueden utilizarios (biodegradación).

b) Tecnología: producción masiva de PVC (cloruro de polivinilo) y PHB (polyhydroxybutirato), un producto que es completamente degrado por microorganismos y producido por $\mathrm{ICI}$, bajo nombre comercial de Biopol.

c) Economía y ciencias sociales: las implicaciones de la producción de PVC y PHB (ésta es mucho más cara que la de PVC; los plásticos son parte del ciclo natural del carbono).

d) Impacto: necesidades ecológicas versus condiciones económicas bajo los condicionantes que supone el desarrollo sostenible (lugares y países donde la producción se lleva a cabo).

Obviamente todos los temas de contenidos no pueden ni deben ser tratados con la misma profundidad, pero la unidad proporciona la oportunidad de interrelacionar aspectos de los diferentes campos y ciencias, conectando, por ejemplo, contenidos de química y biología con tecnología, tomando en cuenta cuestiones de tipo ambiental y económico, así como de tipo social (emplaza. miento de vertederos o plantas de reciclaje de basuras, etc). Se propone también la discusión de la relación entre la ciencia y la tecnología en el descubrimiento y aplicación de conocimientos e innovaciones, discutiéndose también el sentido e impacto de éstas, en términos del desarrollo sostenible.

Los otros dos puntos mencionados más arriba, valores y procedimientos, han sido incluidos en esta unidad, bien como pequeños comentarios sugerentes en el texto, 0 como cuestiones al final de la información que suministra que es en forma de breve relato, junto con el glosario y las referencias bibliográficas, que mayoritariamente hacen referencia a artículos de la revista de investigación/divulgación (New scientist) de fácil acceso tanto para alumnos como profesores.

En el apartado de las cuestiones, se abordan muchos tipos de temas. Por un lado, aquéllos que hacen referencia a contenidos que aparecen formando parte de otras preguntas y son las menos numerosas. Las que más se encuentran son las que adoptan la forma de supuestos de la vida real y que proponen la realización de un experimento que el estudiante debe diseñar para comprobar la o las hipótesis que ha emitido al respecto de ese supuesto. Otras tienen por objetivo la explicación de determinadas decisiones para las que es necesario manejar 
factores de tipo medio ambiental y económico, por ejemplo: «8.d. Explica por qué la producción del plástico Biopol sería una industria adecuada para países en vías de desarrollo.»

El desarrollo de valores como la protección de la naturaleza o la conservación de la biodiversidad se plantea en supuestos que pueden ser desarrollados por los profeso. res y alumnos de formas muy distintas, haciendo especial énfasis en lo que se considere más adecuado en el contexto particular, tales como: «I. Imagina que cientos de bolsas de un plástico biodegradable han sido vertidas

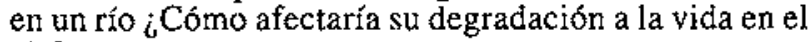
río?»

Supuestos como el que se menciona a continuación: «7. Los médicos saben hoy en día que los huesos se fortalecen y su crecimiento se estimula con corrientes eléctricas. ¿Qué ventajas presentan las agujas y placas de Biopol frente a las metálicas?» se prestan al đesarrollo del concepto de innovación tecnológica además de poder formular múltiples preguntas tanto de biología como de física asociadas a ello.

\section{La formación de profesores}

La naturaleza del concepto de desarrollo sostenible incide directamente con la idea anteriormente expuesta de que la educación está siempre relacionada con el futuro, porque obliga a planificar y a tener en cuenta tiempos diferentes que alargan de forma significativa esa idea de futuro inherente al concepto de educación. La otra idea anteriormente introducida es la necesidad de evaluar no sólo el impacto de las distintas políticas que afectan a los recursos disponibles sino también la actuaciones de los individuos (demografía, etc). En nuestra opinión ambos aspectos son sumamente importantes para la formación de profesores.

Muchos trabajos de investigación muestran que el cambio del currículo es una tarea difícil y compleja y que los profesores juegan un papel muy importante en ella (House, 1979). Numerosos temas pueden ser estudiados desde la perspectiva de lo que es y cómo se produce el cambio, pero la profesionalidad de los profesores es sin duda uno de ellos. Estudios realizados en diferentes países enfatizan aspectos claves, muchos de ellos similares, tales como que las comuridades de profesores suelen ser comunidades cerradas cuyo nivel de conexion e intercambio con el exterior - como con la comunidad científica, con el mundo de las empresas, etc.- es pequeño (Atkin, 1994). También parece que, durante un cierto periodo, al principio de su vida profesional, tienden a reproducir las estrategias de enseñanza/aprendizaje que fueron usadas con ellos por algunos profesores. Reproducción de ideas, valores y conocimientos han sido, hasta hace relativamente poco tiempo, un objetivo importante de los sistemas educativos. Recientes investigaciones muestran que la tendencia de los profesores es apoyar los principios de reformas pasadas, mostrando resistencia a cambiar (Haenisch, 1986, p. 33).
Actualmente, cada vez un mayor número de investigadores apoya la idea de la necesidad de estudiar aspectos del cambio por parte de los profesores, porque como Handy (1989) apunta que el cambio que se está produciendo tiene connotaciones diferentes al que estábamos acostumbrados hasta ahora. Durante años, la idea del cambio se ha asociado a cambio continuo; esto le hacía confortable, porque el pasado servía de guía para un futuro que era previsible. El cambio era «más de lo mismo», probablemente proyectándose hacia situaciones mejores; esta forma de cambiar era esperada y bienvenida. El cambio que actualmente tiene lugar ha sido descrito por Handy (1989) como de tipo discontinuo, que resulta fácil de entender con un símil muy gráfico que tomo de él mismo. Éste nos dice quue el cambio actualmente es equiparable a lo que le sucede a un pequeño sapo al que colocamos en un recipiente con agua que vamos calentando muy lentamente, hasta que comienza a hervir y entonces el sapo muere. Se sentía tan confortable con el cambio lento y continuo que iba experimentando, que al final se alcanza un punto en la temperatura del agua que resulta fatal para él.

Existe un momento en que la variación que se está produciendo presenta una discontinuidad, requiere un cambio de comportamiento, porque se producen situaciones que no permiten ser vividas con los mismos parámetros que anteriormente se utilizaban. Hoy en día las cosas comienzan a ser sustancialmente distintas; la capacidad de predecir el futuro se hace ahora muy incierta.

El cambio de tipo discontinuo requiere una forma de pensar distinta, de tal forma que es necesario no descartar a priori ideas que en situaciones anteriores podían parecer poco adecuadas. Démosles una posibilidad, porque el futuro no será una mejora del pasado sino algo distinto. Este tipo de cambio presenta problemas pero tambien oportunidades. Por otro lado, los períodos de discontinuidad, cuando la experiencia pasada no es útil, cuando no hay reglas o manuales que seguir, si se saben manejar, son justamente en los que se produce crecimiento personal.

Estos argumentos enfatizan la relevancia de tratar sobre el cambio con aquéllos que de forma significativa contribuirán a la configuración de las nuevas generaciones, porque, en períodos de cambio discontinuo, intentar vías nuevas y alternativas de actuar y conocer el impacto de estas acciones se convierte en algo obviamente necesario.

En este sentido, la evaluación del impacto se convierte en una tarea importante de la formación de profesores, para la cual se les debe formar específicamente. Ésta debe ser entendida, por tanto, en un sentido genérico, dado que puede y debe aplicarse, como ya hemos mencionado anteriormente, a una multiplicidad de situaciones a muy distintos niveles, y con distintas características, que van desde la evaluación de las actuaciones individuales (en el ámbito profesional) hasta las colectivas (aquéllas que se proyectan con alcance polftico, para conocer el impacto que están produciendo, de tal forma 
que pueden ser modificadas en caso de que produzcan efectos no deseados). El objetivo es, pues, dar un peso real a aquello que corresponde al efecto que causa las actuaciones, sin por ello quitar la importacia a la planificación, sino centrándose en cómo ésta se lleva a cabo y cuáles son las consectuencias que se van produciendo a lo largo del proceso y en los efectos finales que se obtienen. Es frecuente centrarse en los impactos de las políticas cuando éstas han sido ampliamente implantadas y, sin embargo, lo que la idea de desarrollo sostenible conlleva es la evaluación de los efectos propios y colaterales a lo largo de su implementación para poder estudiar las causas y condiciones que en los contextos particulares se van produciendo, así como aquellos efectos que se generan por la actividad y actuaciones de los colectivos humanos. Es, por tanto, a estos tres niveles de actuación a los que es necesario aplicar la idea de la evaluación del impacto.

Aquí el término evaluación debe ser entendido no tanto como un concepto de tipo técnico, que pueder ser mecánicamente utilizado, sino como un modo de conocimiento flexible capaz de aplicarse a diferentes niveles profesionales, políticos y sociales. Las características que presentan los modelos formativos que asumen este tipo de aprendizaje, que genericamente reciben el nombre de formación-acción, se derivan directamente de la idea de acción y se concretan en que:

- Los estudiantes se vean implicados en situaciones reales, no en situaciones de simulación, sino en aquéllas que, o bien ejemplifiquen los conceptos que se quieren introducir (por ejemplo, la negociación si éste es el tema que se está tratando), o bien a través de la problemática surgida de casos y situaciones que se dan en el propio contexto;

- el planteamiento de las situaciones objeto de estudio permita una perspectiva holística de éstas;

- el estudiante tenga que tomar decisiones barajando elementos tanto de conocimiento, como técnicos, profesionales y también éticos;

- haya que ejercitar la evaluación de la acción realizada, no s6́lo como un ejercicio de autoevaluación individual, sino de forma que pueda ser discutido, tanto con el profesor/tutor como con otros compañeros, para que se realice un análisis crítico que permita valorar el impacto de la propia actuación profesional, posibilitando así que se produzca un debate con argumentos tanto teóricos como prácticos sobre la bondad y oportunidad de las actuaciones y obteniendo algunas ideas que conduzcan a una nueva reestructuración cara a futuras actuaciones.

La evaluación del «impacto» debe entenderse, pues, a distintos niveles y eso hace que requiera de un tratamiento específico en el que aquí no corresponde entrar, pero que, dado que tiene una vertiente concreta para el planteamiento de formación expuesto, parece conveniente ejemplificarla en este sentido. En términos formativos, para que la evaluación se pueda desarrollar, parece adecuado.organizar el ejercicio a través de estrategias de trabajo en pares, en Ias que, a veces, se juega el papel protagonista y, otras, de observador externo de la implementación y de compañero en la planificación y evaluación. Los datos recogidos sobre la implementación de la práctica profesional ejercida es la base del informe que se emitirá sobre el ejercicio profesional. Este informe debe someterse a discusión entre los colegas para hacer un análisis crítico de tal forma que el impacto que la actuación del profesor ha causado pueda ser entendido. Son, tanto los argumentos críticos como aquéllos que apoyan las actuaciones realizadas, los que deben servir como base para introducir modificaciones en las futuras actuaciones.

Estos modelos de formación conducen a la adquisición de conocimiento relevante y útil al mismo tiempo que se desarrolla competencia profesional cuando ésta es defínida como la capacidad de actuar inteligentemente en situaciones sociales complejas e impredecibles que requieren de una forma específica de actuación que sólo es posible in situ. La práctica es, así, una vía de aprender; y el desarrollo y aprendizaje profesionales, una dimensión de la práctica más que una actividad fuera del trabajo (Elliott, 1980).

Concretando la idea de evaluación del impacto, aplicada a la formación de profesores que presento, se puede formalizar de forma escueta como un ejercicio que se desarrolla en sucesivas fases, a saber:

- Estudio de las características de la situación donde se ubica la actuación profesional para la que se produce la formación, que incluye lo que habitualmente se denomina estudio del contexto, entendido éste de forma amplia, no restrictiva y extendiendo los límites hasta lo que sea coherente con el nivel al que se vaya a producir la función de los que están siendo formados, y la naturaleza del rol profesional (conocimiento y competencia).

- Planificación de la actuación que se va a llevar a cabo a modo de protocolo organizativo.

- Implementación del plan trazado, sobre la que se producirá una recogida de datos de diferentes fuentes.

- Evaluación de la acción, incluyendo no sólo valoraciones personales sino datos de terceros, recogidos en todas las fases anteriores. Es en esta fase de donde debe emerger la naturaleza del aprendizaje profesional.

- Todos los contenidos que deban ser estudiados se centran en torno a la práctica, en torno de la cual organizamos, segun el nivel de los estudiantes, la información teórica que se presenta.

Se puede encontrar en la bibliografía, cada vez de manera más insistente, refiriéndose a formación de profesorado, una variante bastante específica que es lo que autores como Stenhouse, Elliott, Ebbutt, entre muchos otros, han dado en llamar investigación-acción y que durante años ha sido centro de polémica debido justamente al término investigación que se utiliza. En mi opinión ésta introduce quizás lo más específico e interesante de la 
propuesta y es la definición, al comenzar el ciclo formativo, de un aspecto que por motivos distintos, interesa estudiarse y que puede ser tan extenso como se considre oportuno, desde un currículo experimental a un aspecto de una estrategia de enseñanza-apredizaje concreta, cuyo impacto quiera conocerse.

\section{EI cambio es un proceso de aprendizaje}

Planteado de esta manera, se puede entender que la teoría implícita en el cambio es una teoría de aprendizaje, puesto que éste se encuentra en el mismo corazón del cambio. Para muchos autores aprender es un ciclo que incluye múltiples actividades. Schön (1983), por ejemplo, plantea el aprendizaje en una circularidad doble, se aprende al resolver un problema concreto, pero también sabiendo cómo éste se resolvió. De hecho, ésta última consiste, en su opinión, en entender cómo se ha aprendido a resolverlo e introducir el conocimiento de cómo aprendemos en el bagaje cotidiano.

Subyacente a todo lo anteriormente planteado se encuentra la afirmación de que el aprendizaje más eficaz es aquel que sucede en las situaciones reales, con personas y problemas reales, más que a través de recitar nuevos hechos y conocimientos descontextualizados. Hace ya muchos años que Dewey (1938) planteó el aprendizaje como un proceso de «descubrimiento personal», idea opuesta completamente al pensamiento de Skinner, que planteaba el aprendizaje como «entrenamiento», aprendizaje éste que no se sustenta en la existencia de un cambio, sino sencillamente en la repetición. Por el contrario, en el primer caso, el aprendizaje real comienza cuando nos formulamos una pregunta o intentamos resolver un problema. Si no hay pregunta, no hay respuestas. Si las preguntas no son propias o bien se olvidan, cosa que sucede en muchos casos, la contestación está encaminada a responder lo que Ia fórmula quiere oir.

Figura 1

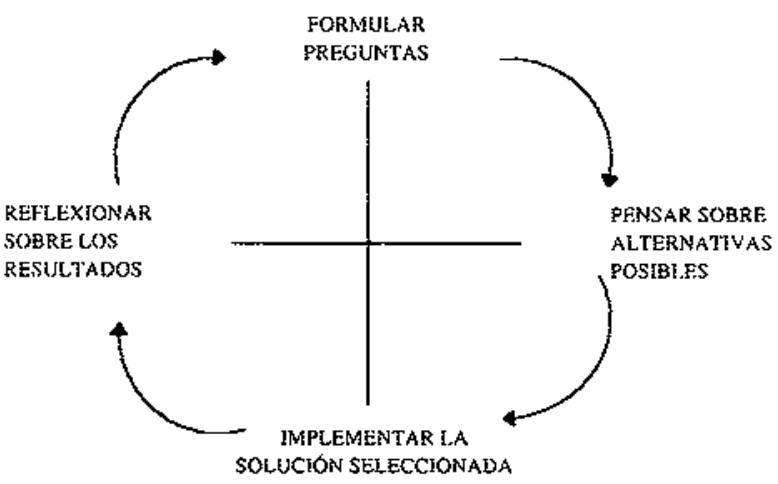

El aprendizaje se puede interpretar como una estructura circular, como una rueda (Fig. 1) con cuatro ejes que puede y debe girar. Se parte de una serie de preguntas en el momento adecuado para las que se ensayan respuestas sobre las que se reflexiona, to que puede conducir a nuevas preguntas. El problema consiste en que en muchas ocasiones la rueda de los adułtos está parada o gira muy despacio, pero basta con mirar a un niño pequeño para ver que la rueda puede girar muy velozmente.

La rueda puede empezar a girar cuando nos formulamos una pregunta, o asumimos un reto, o queremos resolver un problema. Habitualmente nos encontramos con que existen varias respuestas, por eso es necesario pensar sobre las distintas posiblidades que se nos ocurren, también en las teorías que están implicítas en el hecho que observamos. Estamos en el momento de la especulación, de la reorganización de la pregunta, es el momento de pensar libremente, el momento de buscar claves. Nunca, ni las ideas que se nos ocurran, ni las teorías que se revisen serán demasiadas. Estamos en la segunda etapa de la rueda, la de emitir propuestas distintas, pensar en cómo resolver el problema. Pero estas alternativas posibles deben probarse en la realidad para saber la que es viable; algunas lo son, otras no. Ésta es la tercera etapa de la rueda. La ưltima etapa es la reflexión final.

Si sólo con resolver el problema se da el ejercicio por terminado, no se habrá producido aprendizaje con el sentido que se está planteando. El cambio sólo se produce cuando sabemos qué es lo que ha sucedido, por qué se ha resuelto el problema. La etapa de la implementación tiene muchos partiarios, los pragmáticos, las personas de acción, cuya reacción inmediata frente a un problema es empreder su solución con las herramientas o argumentos que tienen a su alcance, en los que no hay tiempo para pensar sobre las teorías implicítas. A menudo, cuando se solucionan a la primera las preguntas intciales, no se repara en el ejercicio realizado para obtener la solución; lo que con frecuencia conduce a insistir una vez tras orra probando distintas alternativas, sin que se sepa por qué no dan resultado las ya probadas.

Afirmamos así que aprender no es sólo conocer respuestas, ni es sólo estudiar o probar, ni se puede medir por los exámenes tradicionales que se centran en los aspectos teóricos que se recuerdan; ni es automático, sino que requiere una gran cantidad de energía y apoyo, es una especie de crecimiento personal. Aprender no es sólo buscar y encontrar lo que otros ya saben, porque, tal y como lo planteamos, es algo que forma parte de las vidas de los individuos y, por lo tanto, se centra en los retos que se presentan a cada uno y que se deben resolver de forma particular.

La teoría implícita en este proceso de aprendizaje es el «aprendizaje situado», propuesto por Brown (1989) y otros colegas, como un nuevo paradigma alternativo al paradigma del procesamiento de información. En él se argumenta que los siguientes aspectos de la cognición diaria son esenciales para toda persona cuando aprende: resolviendo dilemas que emergen y no resolviendo puzles predefinidos; utilizando la planificación como un recurso para contextualizar las actuaciones más que como planes prescriptivos para la acción; y entendiendo y tratando los significados como entidades socialmente 
negociadas en contextos específicos más que cómo objetos preexistentes de codificación, trasmisión o descodificación. Los puntos centrales del aprendizaje situado se pueden resumir en:

a) la cognición entraña una "conversación» del individuo con las situaciones;

b) el conocimiento implica una relación de acción práctica entre la mente y ei mundo; y

c) el aprendizaje entraña un ejercicio cognitivo en la realización de actividades múltiples, algunas de ellas, colaborativas.

En el paradigma del procesamiento de la información, los planes se sitúan en el centro del proceso de enseñanza-aprendizaje. Cuando la estrategia de enseñanza está embebida en los planes, la enseñanza se entiende genéricamente como un proceso de información que ajusta estructuralmente con el proceso cognitivo del que aprende a través de estímulos del medio. La estrategia de enseñanza (Sáez, 1993) garantiza, entonces, cualquier cambio que se produce en las estructuras cognitivas del que aprende. La interacción del estudiante con el plan propuesto en el paradigma cognitivo se restringe, pues, al concepto de la ciencia física de «acción recíproca o influenciam, negando además la historicidad de la situación de aprendizaje (Stribel, 1989).

Por ello la cuestión central es: ¿pueden los estudiantes aprender en una situación en la que deben actuar como si construyeran y manipularan símbolos que ya han sido definidos en un plan preespecificado? Se puede argumentar que todas la situaciones reales de aprendizaje implican una cierta relación con el contexto, la acción situacional, y no con una interacción especificada en un plan. La construcción social de la realidad se fundamenta en cada cognición situacional y en la práctica, y los planes pueden ser una respuesta a dilemas emergentes configurados por situaciones históricas y acciones. Entendidos así los planes, son recursos para las actuaciones prácticas, para comunicar acciones a otros y para

\section{REFERENCIAS BIBLIOGRÁFICAS}

ANDERSON, B. (1994). On knowing through integration. Unpublished paper. Suecia: University of Göteborg.

ATKIN, M. (1994). Paper presentado en la Conferencia Science Curriculum and scientific literacy in EU countries. (Proxima publicación.)

BLACK, P. (1993). The Purpose of Science Education, en Whitelegg, E. et al. (eds.). Challenges and Opportunities for Science Education. Londres: Chapman.

BROPHY, M. (1991). Global Science. School Science Review, $73(262)$, pp. 59-66. reflexionar sobre la reconceptualizaciones de las acciones situacionales. En el proceso de enseñanza-aprendizaje, cuando un profesor o un alumno se detiene en los detalles, la teoría y los planes se abandonan y se recurre a las destrezas que están embebidas en la situación e interiorizadas. De esta manera, la realidad de la enseñanza y el aprendizaje termina siendo una construcción contextualizada y particular de los implicados.

\section{CONCLUSIONES}

La planificación del currículo debe hacerse a más largo plazo para intentar un equilibrio entre las decisiones de hoy y su impacto futuro. El currículo que se recomiende no podrá presentar la relevancia de sus temas en función de la propia disciplina científica sino más bien orientados a posibilitar y capacitar a los estudiantes a desenvolverse y tratar con un problema relevante, como los que relacionan ciencia y tecnología, tomando en consideración cuestiones de tipo ambiental y analizando el impacto de las innovaciones en función del planteamiento de desarrollo sostenible. El enfoque "ciencia, tecnología y sociedad" parece ser el más adecuado siempre y cuando la $S$ de sociedad se entienda como el impacto en el medio y en la economía, en términos sociales, éticos y globales, con el objetivo de actuar localmente.

El impacto debe ser evaluado: no sólo ta elección de los temas en su idoneidad para capacitar a los estudiantes a tratar con problemas relevantes, sino que la evaluación de las destrezas profesionales debe ser una parte esencial de la formación de profesores y debe introducirse en las actividades de formación permanente. El despiazamiento del paradigma del aprendizaje como procesamiento de información a un enfoque de «aprendizaje situado»se propone como la teoría implícita de las estrategias de enseñanza-aprendizaje para que el concepto de desarrollo sostenible se difunda en el mundo real.
BROWN, J.S. (1988). Steps Toward a New Epistemology of Situated Learning, en Proceedings of the ITS-88. International Conference on Intelligent Tutoring Systems. Montreal: University of Montreal.

CROSS, R.T. (1993). The Risk of Risks: A Challenge and a Dilemma for Science and Technology Education. Research in Srience and Technology Education, 11(2), pp. 171-183.

DEWEY, J. (1938), Experience and Education. Collier MacMillan Publishers. 
ELLIOTT, I. (1980). Adult Learning and Development: Implications for In-Service Teacher Education. París: OECD.

HAENISCH, H. (1986). Wirkungen offener Lehrplanarbeit. Soest (Arbeitsberichte zur Curriculumentwicklung, 9).

HALE, M. (1993), Sustainable Development Through Environmental Technology: Education and Training Requirements, en Global Fortm on Environmental Education for Sustainable Development. Nueva Delhi, India: Indian Environmental Society.

HANDY, C. (1989). The Age of Unreason. Londres: Arrow Books.

HOLBROOK, J. y CHISMAN, D. (1990). The Future Direction of Sustainable Development in the Curriculum, en Penick, J.E. y Stiles, J.R. (eds.) Sustainable Development Development for aNew World Agenda. Winnipeg, Canada: STAM/CASE/ ICASE.

JALAL, K.F. (1990). Nineties: The Decade of Challenges and Opportunities en Bandhu, D. et al. (eds.). Environmental Education and Sustainable Development. Nueva DeIhi, India: Indian Environmental Society.

KORTLANI), K. (1992). Environmental Education: Sustainable Development and Decision Making, en Yager, R.E. (ed.). The Stutus of Science-Technology-Society Reform Efforts Around the World. Petersfield: ICASE.

MARATHE, E. (1994). National and Global Responsibilities Towards Sustainable Development, en Proceedings of the 6th IOSTE Symposium on World Trends in Science and Technology Education. Palm Springs, EE.UU. (En prensa).
MEADOWS, D. (1972). The Limits to Growth. Nueva York: Universe Books.

NACHTIGALL, D. (1988). New Priorities in Science and Technology Education, en Riquarts, $\mathrm{K}$. (ed.). Science and Technology Education and the Quality of Life, Vol. III. Kiel: Institute for Science Education (IPN).

POWER, C. (1990). Policy Issues in Science Education: An International Perspective, en Jenkins, E. (ed.). Policy Issues and School Science Education. Leeds: Centre for Studies in Science and Mathematics Education, Leeds University.

RIQUARTS, K. (ed.) (1987). Science and Technology Education and the Quality of Life, Vol. II: Technology Education/ Science-Technology-Society. Kiel: Institute for Science Education (IPN).

SÁEZ, M. (1993). New Trends in the Teaching and Learning Strategies in Science Education for the Year 2000, en Intermational Forum on Scientific and Technological Literacy for All (Project 2000+). París: UNESCO (ED-93/CONF. 016.REF1.3).

SCHÖN, D.A. (1983). The Reflective Practitioner. Nueva York: Basic Books.

STRIEBEL, M.J. (1989). Instructional Design and Situated Learning: Is a Marriage Possible? Paper presented at the AERA Conference, San Francisco.

UZWYSHYN, E. (1990). Principles of Sustainable Development, en Penick, J.E. y Stiles, J.R. (eds.). Sustainable Development for a New World Agenda. Winnipeg: STAM/CASE/ICASE.

YAGER, R.E. (ed.) (1992). The Status of Science-rechnologySociety Reform Efforts Around the World. Petersfield: ICASE.

[Artículo recibido en junio de 1994 y aceptado en septiembre de 1995.] 